\title{
MHD Mixed Convection Flow in a Lid Driven Enclosure with a Sinusoidal Wavy Wall and a Heated Circular Body
}

\author{
Kakali Chowdhury ${ }^{1}$, Abdul Alim ${ }^{2}$ \\ ${ }^{1}$ Department of Electrical \& Computer Engineering, Presidency University, Dhaka, Bangladesh \\ ${ }^{2}$ Department of Mathematics, Bangladesh University of Engineering \& Technology, Dhaka, Bangladesh
}

Email address:

kakalishome@yahoo.com (K. Chowdhury), maalim@math.buet.ac.bd (A. Alim)

To cite this article:

Kakali Chowdhury, Abdul Alim. MHD Mixed Convection Flow in a Lid Driven Enclosure with a Sinusoidal Wavy Wall and a Heated Circular Body. International Journal of Fluid Mechanics \& Thermal Sciences. Vol. 5, No. 4, 2019, pp. 102-110. doi: $10.11648 /$ j.ijfmts.20190504.13

Received: August 19, 2019; Accepted: October 28, 2019; Published: November 6, 2019

\begin{abstract}
MHD mixed convection flow in a lid driven enclosure with a sinusoidal wavy wall and heated circular body located at the center of the enclosure is studied numerically using finite element analysis. The upper wall is moving with a uniform velocity by unity, and other walls are in no slip condition. The enclosure vertical walls are insulated while the heated circular body inside the enclosure is maintained at a uniform temperature higher than the upper wall and lower wavy surface. Calculations are carried out through solving governing equations for different parameters by using finite element method. The investigation is conducted for various values of Richardson number $R i$ and Prandtl number $P r$. The heat transfer characteristics and flow pattern inside the enclosure are presented in the form of streamlines and isothermal contours. Heat transfer rate in terms of the average Nusselt number and average fluid temperature inside the enclosure are presented for different parameters. The results indicate that the average Nusselt number at the heated surface and average temperature of the fluid inside the enclosure are strongly dependent on the configuration of the system under different geometrical and physical conditions.
\end{abstract}

Keywords: MHD Mixed Convection, Finite Element Method, Square Enclosure, Circular Heated Body

\section{Introduction}

Mixed convection flow in any enclosure occurs due to combined natural and forced convection flow. Natural convection occurs due to buoyancy flow produced by thermal non-homogeneity within the enclosure or enclosure walls and forced convection occurs due to shear flow caused by the movement of any wall in the enclosure. The study of mixed convection flow in lid driven enclosure has received a massive attention from the researchers due to its vast application in the technological sector. Such applications include cooling of electronic devices, heat exchangers, drying technologies, food processing, and metal coating and casting, flow and heat transfer in solar ponds etc. There are many investigations on mixed convective flow in lid-driven cavities. Many different configurations and combinations of thermal boundary conditions have been considered and analyzed by various investigators.

Saha et al [1] investigated numerically mixed convection heat transfer in a lid driven enclosure with wavy bottom surface. They found that Reynolds number, Grashof number and the number of undulations of the wavy surface have significant effect on the flow fields, temperature distribution and heat transfer in the enclosure. Nasrin [2] investigated mixed magneto-convection in a lid driven enclosure with a sinusoidal wavy wall and a central heat conducting body. She considered a heat conducting square body located at the center of enclosure. The enclosure horizontal walls are perfectly insulated while the corrugated right vertical surface is maintained at a uniform temperature higher than the left lid. She found that the influence of $\mathrm{Ha}$ does not affect significantly the thermal current activities. But the flow pattern changes dramatically owing to the hindrance of the imposed magnetic field. Rahman and Alim [3] investigated MHD mixed convection flow in a vertical lid driven square enclosure including a heat conducting horizontal circular cylinder with Joule heating. They reported that mixed convection parameter $R i$ affects significantly on the flow structure and heat transfer inside the enclosure and the 
overall heat transfer decreases with the increase of joule heating parameter $J$. Rahman et al. [4] studied finite element analysis of mixed convection in a rectangular cavity with a heat conducting horizontal circular cylinder. They found that the increasing Prandtl number increases the average Nusselt number at the hot wall and decreases the average temperature of the fluid in the cavity. They also showed that the average Nusselt number at the heated surface is always the highest and the average temperature of the fluid in the cavity is the lowest for the large value of Re. Oztopet al [5] investigated MHD mixed convection in a lid driven cavity with corner heater. They showed that heat transfer decreases with increasing of Hartmann number and the rate of reduction is higher for high values of the Grashof number. YadollahiFarsani and Ghasemi [6] investigated magnetohydrodynamic mixed convective flow in a cavity. They used an enclosure of which lower surface is heated from below whereas other walls of the enclosure are thermally insulated. They found that as Hartmann number increases the Nusselt number, representing heat transfer from the enclosure decreases. Sheikhzadeh et al. [7] investigated effect of a magnetic field on mixed convection of a nanofluid in a square cavity. They found that the rate of heat transfer increases with the increasing value of Reynolds number and it decreases with the increasing value of Hartmann number. Parvin and Nasrin [8] studied the effects of Reynolds and Prandtl number on mixed convection in an octagonal channel with a heat generating hollow cylinder. They found that average nusselt number at the heated surface increases as Re increases and the maximum temperature of the fluid is found optimum for the lowest $R e$ as well as $R i$. They also found that escalating the Prandtl number increases the average Nusselt number at the heated surface and devalues the maximum temperature of the fluid. Bakar et al [9] investigated the mixed convection in lid-driven cavity with inclined magnetic field. They showed that the heat transfer rate indicated by the average Nusselt number increases by increasing the magnetic field angle, increasing the Richardson number leads to a decrease in the heat transfer rate and mixed convection flow is retarded by the presence of the magnetic field. Muthtamilselvan and Doh [10] investigated mixed convection of heat generating nanofluid in a lid-driven cavity with uniform and non-uniform heating of bottom wall. They showed that in the condition of uniform heating the heat transfer rate generally decreases from bottom corners and attains maximum at the center of the bottom wall for all Richardson number. The non-uniform heating condition attains maximum heat transfer rates at the center of the bottom wall than with uniform heating condition for all Richardson number. Oztop et al. [11] investigated MHD mixed convection in a lid driven cavity with corner heater. They showed that magnetic field plays an important role to control heat transfer and fluid flow. Heat transfer decreases with increasing of Hartmann number and the rate of reduction is higher for high values of the Grashof number. Sivasankaran et al. [12] investigated numerical study on mixed convection in a lid driven cavity with non- uniform heating on both sidewalls. They found that the nonuniform heating on both walls provides higher heat transfer rate than non-uniform heating of one wall. They also observed that the heat transfer rate increases as the Prandtl and Reynolds number increase. Roslan et al. [13] investigated natural convection in an enclosure containing a sinusoidally heated cylindrical source. They found that the cylinder temperature oscillation can drastically change the flow and temperature fields. They also observed that heat transfer rate tends to increase by oscillating the source temperature signal. Parvin et al. [14] investigated the analysis of the flow and heat transfer characteristics for MHD free convection in an enclosure with a heated obstacle. They found that the influence of magnetic parameter $\mathrm{Ha}$ on streamlines and isotherms are remarkable. The eddies in the streamlines are reduced and the thermal current surrounding the hot body is thin with elevating $H a$. The average Nusselt number $\mathrm{Nu}$ at the circular body surface is enhanced for larger $R a$ and diameter $D$ whereas devalued with growing $H a$. Rahman et al. [15] investigated a numerical study of mixed convection in a square cavity with a heat conducting square cylinder at different locations. They found that the flow field and temperature distribution inside the cavity are strongly dependent on the Richardson numbers and the position of the inner cylinder.

\section{Mathematical Formulation}

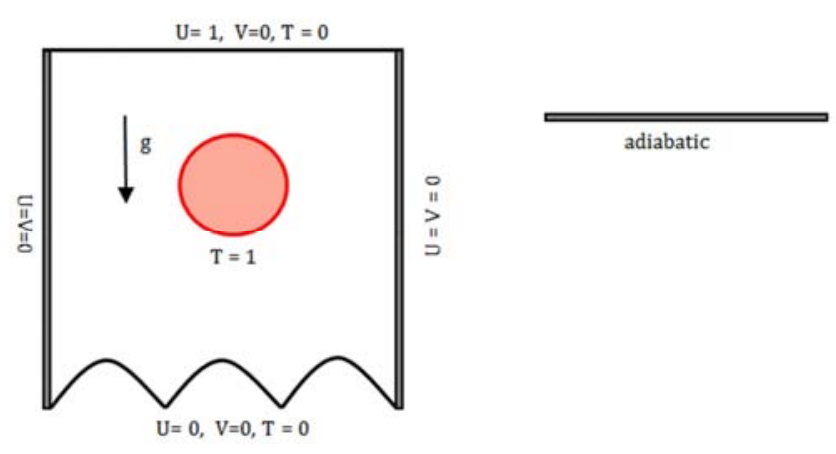

Figure 1. Schematic view of the enclosure.

The numerical procedure used in this work is based on the Galerkin weighted residual method of finite element formulation. The application of this technique is well described by Taylor and Hood and Dechaumphai. In this method, the solution domain is discretized into finite element meshes, which are composed of non-uniform triangular elements. Then the nonlinear governing partial differential equations (i.e. mass, momentum and energy equations) are transferred into a system of integral equations by applying Galerkin Residual method. The integration involved in each term of these equations is performed by using Gauss's quadrature method. The nonlinear algebraic equations so obtained are modified by imposition of boundary conditions. These modified nonlinear equations are transferred into linear algebraic equations by Newton-Raphson iteration. Finally, these linear equations are solved by using Triangular Factorization method. A schematic view of the square 
enclosure with above mentioned boundary conditions is shown in figure 1 . The side length of the enclosure is denoted by L. The upper wall and wavy bottom surface are kept at temperature $T_{c}$ while the vertical walls are kept adiabatic and the circular body is kept at temperature $T_{h}$.

The continuity, momentum and energy equations for laminar, steady state, two dimensional flow in dimensionless form are expressed as

$$
\begin{gathered}
\frac{\partial \mathrm{U}}{\partial \mathrm{X}}+\frac{\partial \mathrm{V}}{\partial \mathrm{Y}}=0 \\
U \frac{\partial U}{\partial X}+V \frac{\partial U}{\partial Y}=-\frac{\partial P}{\partial X}+\frac{1}{\operatorname{Re}}\left(\frac{\partial^{2} U}{\partial X^{2}}+\frac{\partial^{2} U}{\partial Y^{2}}\right) \\
U \frac{\partial V}{\partial X}+V \frac{\partial V}{\partial Y}=-\frac{\partial P}{\partial Y}+\frac{1}{\operatorname{Re}}\left(\frac{\partial^{2} V}{\partial X^{2}}+\frac{\partial^{2} V}{\partial Y^{2}}\right)+R i \theta-\frac{V H a^{2}}{\operatorname{Re}} \\
U \frac{\partial \theta}{\partial X}+V \frac{\partial \theta}{\partial Y}=\left(\frac{\partial^{2} \theta}{\partial X^{2}}+\frac{\partial^{2} \theta}{\partial Y^{2}}\right)
\end{gathered}
$$

The dimensionless variables are defined as:

$$
X=\frac{x}{L}, Y=\frac{y}{L}, U=\frac{u}{U_{0}}, V=\frac{v}{U_{0}}, D=\frac{d}{L}, P=\frac{p}{\rho U_{0}^{2}}, \theta=\frac{T-T_{c}}{T_{h}-T_{c}}
$$

The governing parameters in the preceding equations are the Reynolds number $\mathrm{Re}=\frac{\mathrm{U}_{0} \mathrm{~L}}{\mathrm{v}}$, Richardson number $\mathrm{Ri}=\frac{\mathrm{g} \beta \Delta \mathrm{TL}}{\mathrm{V}_{0}{ }^{2}}$ and Prandtl number $\operatorname{Pr}=\frac{v}{\alpha}$. The shape of the bottom wavy surface has the pattern $\mathrm{Y}=\mathrm{A}(1-\cos 2 \pi \lambda \mathrm{x})$, where $A$ is the dimensionless amplitude of the wavy surface and $\lambda$ is the number of undulations.

The associated dimensionless boundary conditions are as follows:

$P=0 ;$ at all boundaries

$U=V=0$; at the enclosure's adiabatic vertical walls

$\theta=0$; at the wavy wall and upper horizontal wall

$\frac{\partial \theta}{\partial \mathrm{Y}}=0$; at the vertical walls

At the heated circular body surface $U=V=0, \theta=1$

The heat transfer coefficient in terms of the local Nusselt number is defined by $N u=-\frac{\partial \theta}{\partial \eta}$ where $\eta$ denotes the normal direction on a plane and the bulk average temperature in the enclosure is defined as $\theta_{a v}=\int \frac{\theta}{S} d S$.

\section{Numerical Validation}

To validate the present numerical code, the results for MHD mixed convection flow around a heated circular obstacle placed in the centre of the enclosure has been compared with those obtained by Saha et al. The comparison of the results obtained from present code with those of Saha et al is demonstrated for two different Grashof numbers $G r=10^{4}$ and $G r=10^{6}$ at $\operatorname{Re}=10^{2}, A=0.05, \operatorname{Pr}=0.71$ in figure 2. As seen from these figures the obtained results show very good agreement.

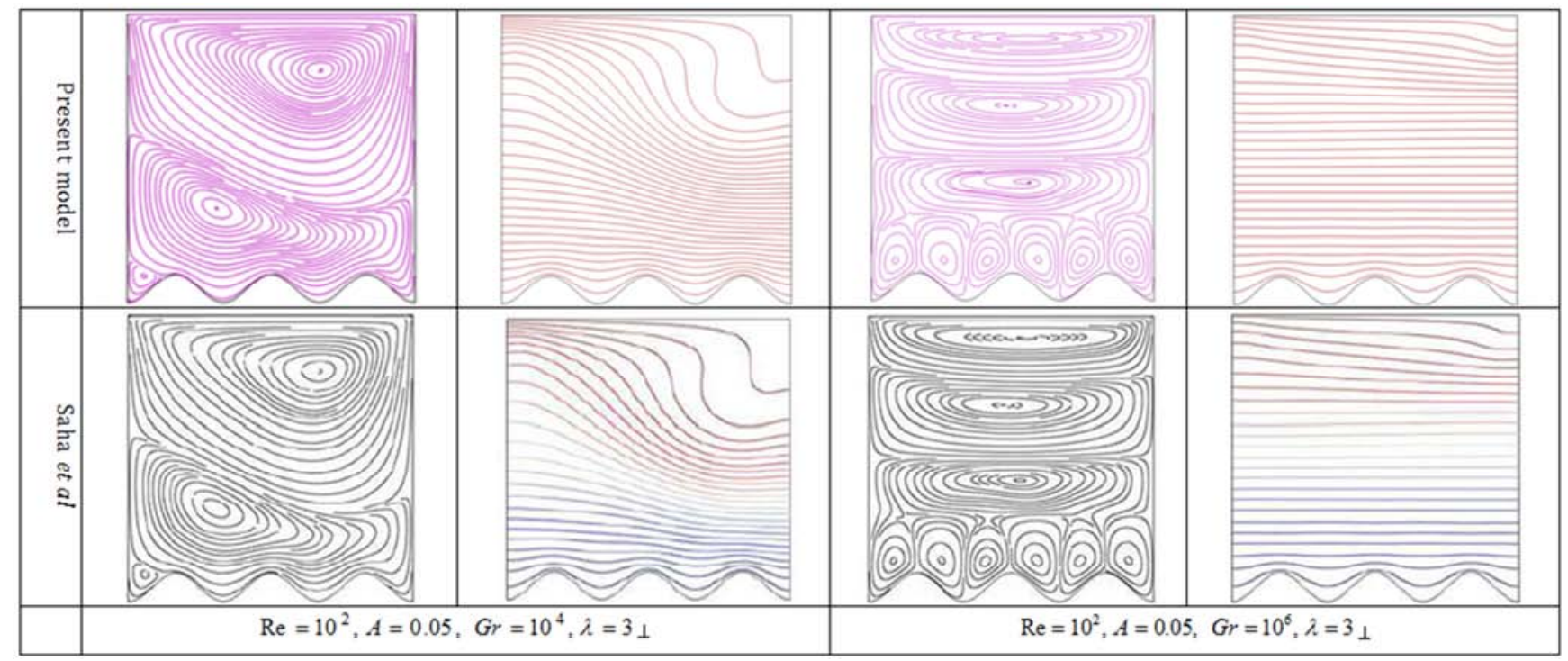

Figure 2. Comparison of streamlines and isotherms between present model (upper) and the model of Saha et al (lower) at Pr=0.71.

\section{Results and Discussion}

The characteristics of the flow and temperature fields in the lid driven enclosure with wavy bottom surface are analyzed for different values of Hartman number $H a$ and Prandtl number Pr. In the present numerical study, the value of the dimensionless numbers is considered as following: $\operatorname{Re}=10^{2}, 1 \leq \lambda \leq 3, D=0.3, A=0.05$ and $0 \leq H a \leq 60, \quad 0.71 \leq \operatorname{Pr} \leq 13.4, \quad 0.1 \leq \operatorname{Ri} \leq 10$. 


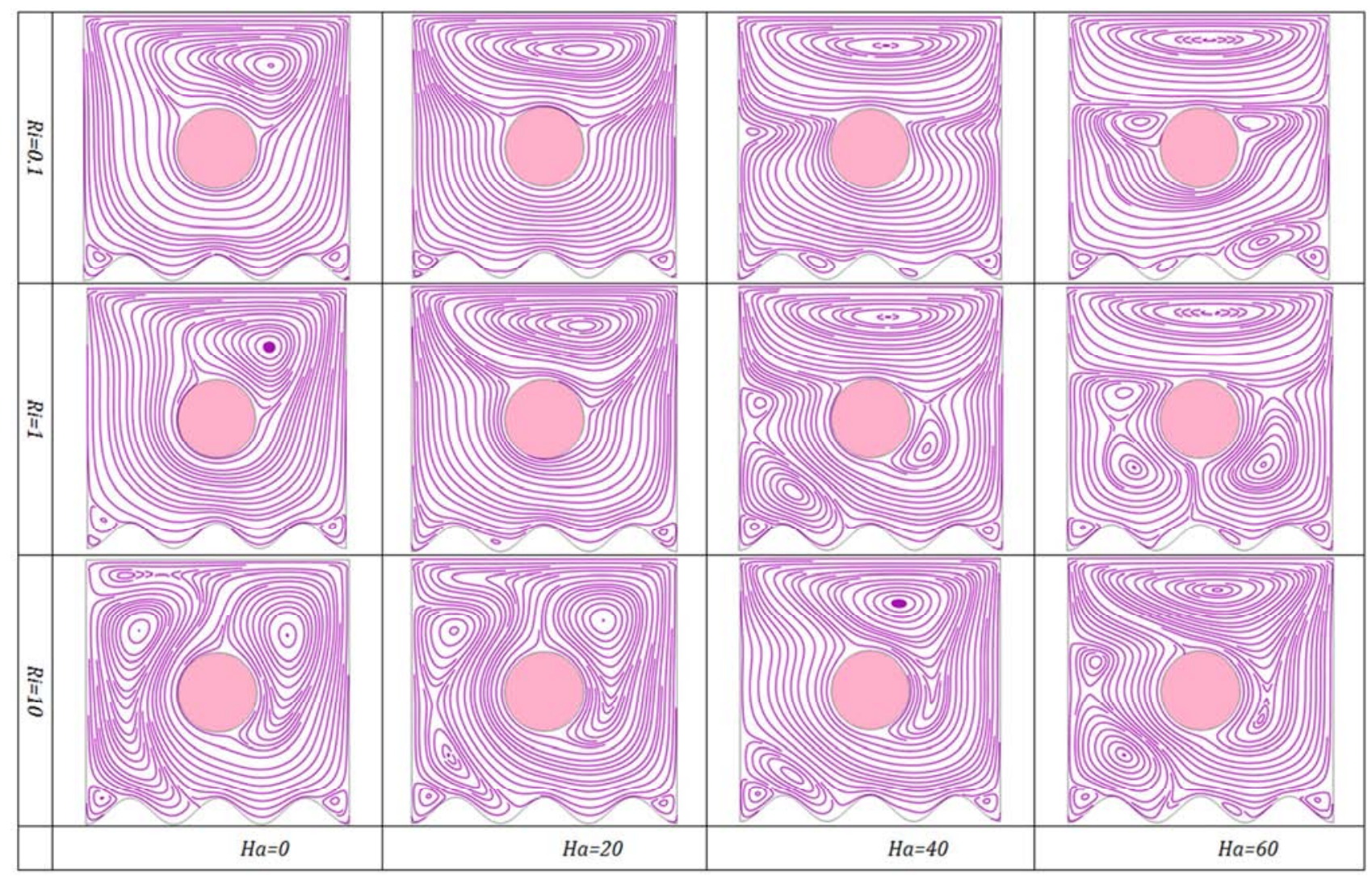

Figure 3. Streamlines for various values of Ha and Ri at $\operatorname{Re}=100, \operatorname{Pr}=0.71, A=0.05$.

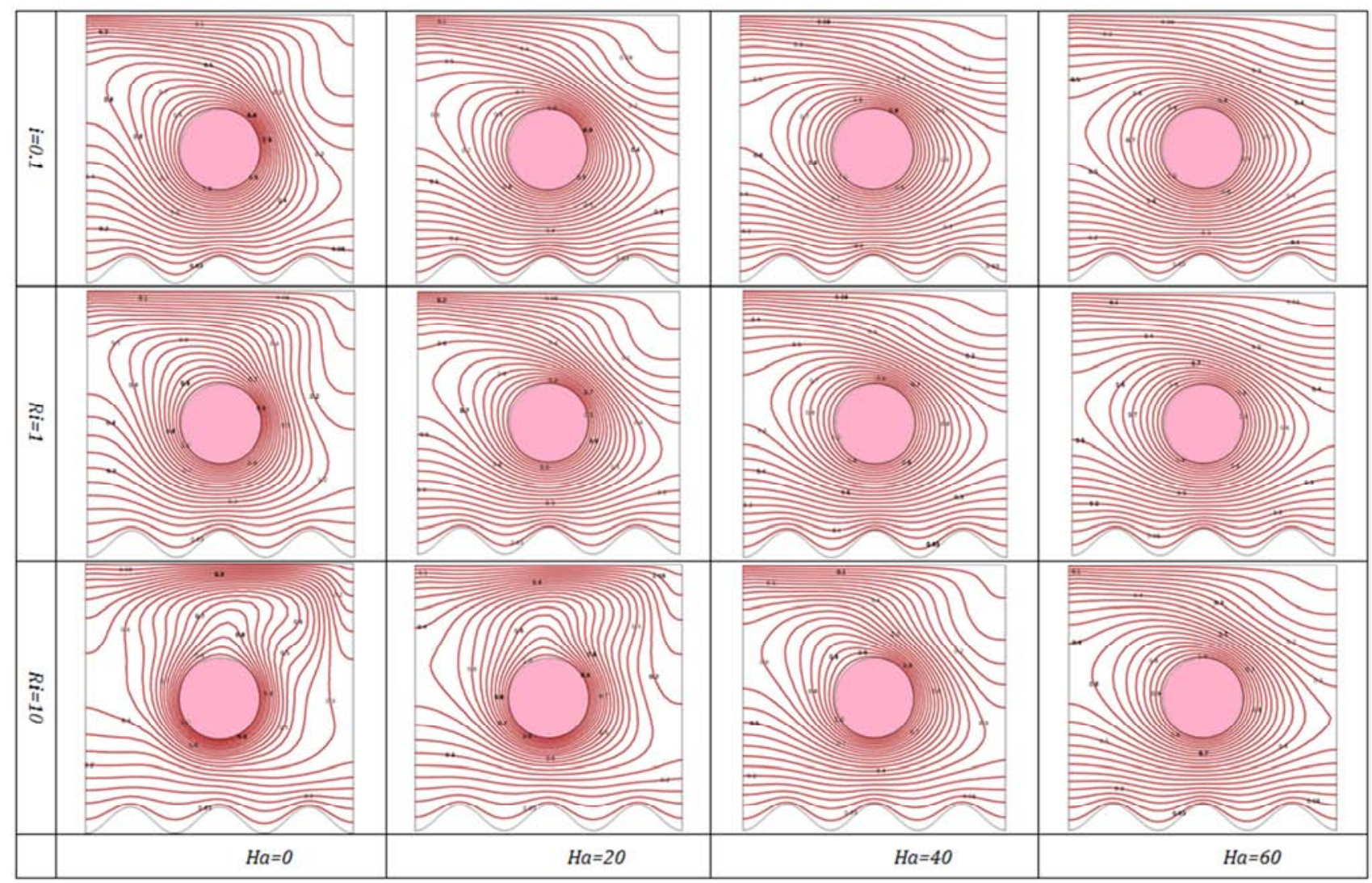

Figure 4. Isotherms for various values of Ha and Ri at $\operatorname{Re}=100, \operatorname{Pr}=0.71, A=0.05$. 
The basic features of flow and heat transfer in the lid driven enclosure are analyzed with the help of streamlines and isothermal contours. Also the average $N u$ along heated surface and average temperature of fluid for various values of $\mathrm{Ha}$ and $\mathrm{Pr}$ are analyzed. The overall features of streamlines for the variation of $H a$ and $R i$ are depicted in figure 3 where $\operatorname{Re}=100, \operatorname{Pr}=0.71, A=0.05$ are kept fixed. At $R i=0.1$ that is the ratio of Grashof number and square of Reynolds number is less than 1 , which means that Richardson number $R i<1$, the forced convection is dominating. In this case, for $H a=0 \mathrm{i}, \mathrm{e}$, in the absence of magnetic field the fluid flow is characterized by a primary rotating unicellular vortex occupying the whole enclosure by the movement of top lid. Upto $H a=20$ the vortex remains unaffected and for higher value of $\mathrm{Ha}$ the core of the vortex is divided mainly into two parts positioned at the top and bottom part of the enclosure.

According to the definition of fluid flow, the buoyancy flow produced by the temperature difference of heated body and different walls of the enclosure balances the flow produced by the moving wall. That is natural convection due to buoyancy force and forced convection due to moving wall both are dominant and hence the total heat transfer in the enclosure is led by mixed convection. In this case, for lower values of $\mathrm{Ha}$ the unicellular vortex remains the same as that of for $R i=0.1$. But for higher values of $H a$ the bottom part of the vortex is divided in three parts. When $R i=10$ according to definition, the flow in the enclosure will be led by free convection flow dominated by buoyancy force. In this case, for $H a=0$, i,e, in the absence of magnetic field the whole enclosure is divided into 2 vortices along the adiabatic walls among which the left vortex is smaller than the right sided vortex. With the increasing value of $H a$ the size of the smaller vortex reduces and the larger one increases.

The corresponding effects on the temperature field are shown in figure 4. At all $R i$, for the highest value of $H a$, the isotherms are almost parallel to the horizontal walls and the circular body, which indicates that most of the heat transfer process is carried out by conduction. However some deviations in the conduction dominated isothermal lines are initiated near the right top surface of the cavity for $\mathrm{Ha}=20$. With the decreasing value of $\mathrm{Ha}$ this distortion decreases. At the same time the distortion of the isothermal lines increases quickly with the increasing value of $R i$ and at $R i=10$ the isothermal lines drastically changed with the decreasing value of $\mathrm{Ha}$. Moreover the formation of the thermal boundary layer near the upper horizontal wall and near the heated obstacle is to be initiated for the lower values $\mathrm{Ha}$. This is owing to the dominating influence of the convective current in the cavity.

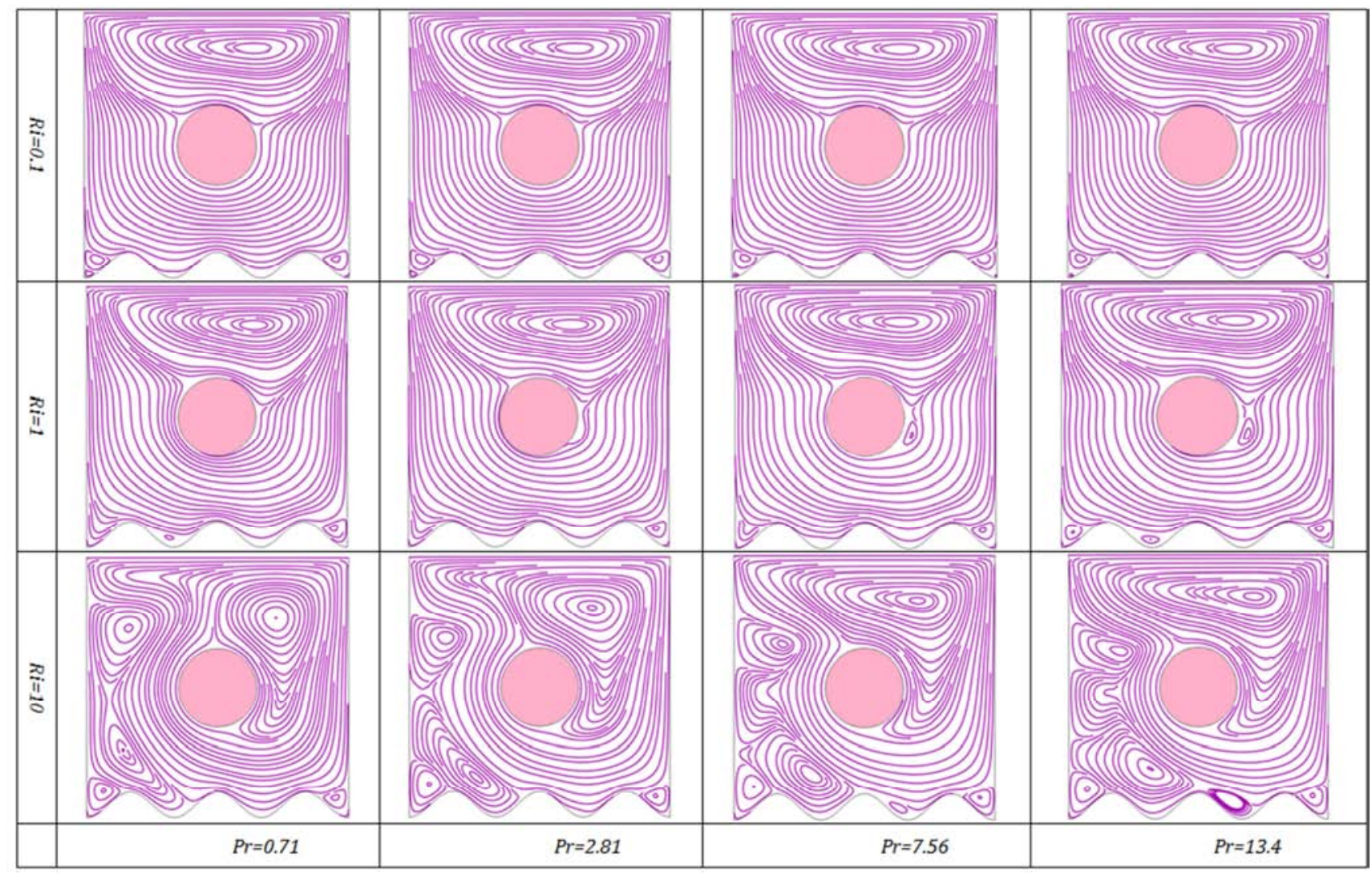

Figure 5. Streamlines for various values of $\operatorname{Pr}$ and $\mathrm{Ri}$ at $\mathrm{Re}=100, H a=20, A=0.05$

The overall features of streamlines for the variation of $\mathrm{Pr}$ and $R i$ are depicted in figure 5 where $\mathrm{Re}=100, A=0.05, \mathrm{Ha}=20$ are kept fixed. At $R i=0.1$ for $P r=0.71$, i,e, when the fluid is considered as air the fluid flow is characterized by a unicellular vortex dividing in two parts just above the heated obstacle occupying the whole cavity. In this forced convection dominated region the streamlines remains unaffected for larger values of $\operatorname{Pr}$. At $R i=1$, that is in mixed 
convection dominated region the characteristics of fluid flow remain almost the same for all values of $\operatorname{Pr}$. At $R i=10$ that is in natural convection dominated region for $P r=0.71$ two vortices are created. Between them the larger one is around the heated obstacle and near the right vertical wall and the smaller one is near the left vertical wall. For higher values of $\mathrm{Pr}$ the core of the larger vortex is displaced near the upper vertical wall and the smaller vortex is divided in little vortices. In the forced and mixed convection dominated region, heat transfer characteristics has no remarkable change with the increasing value of $P r$ but in the free convection dominated region the core of the larger vortex gradually displaced near the upper wall and the smaller vortex has divided in some tiny vortices with the increasing value of $P r$.

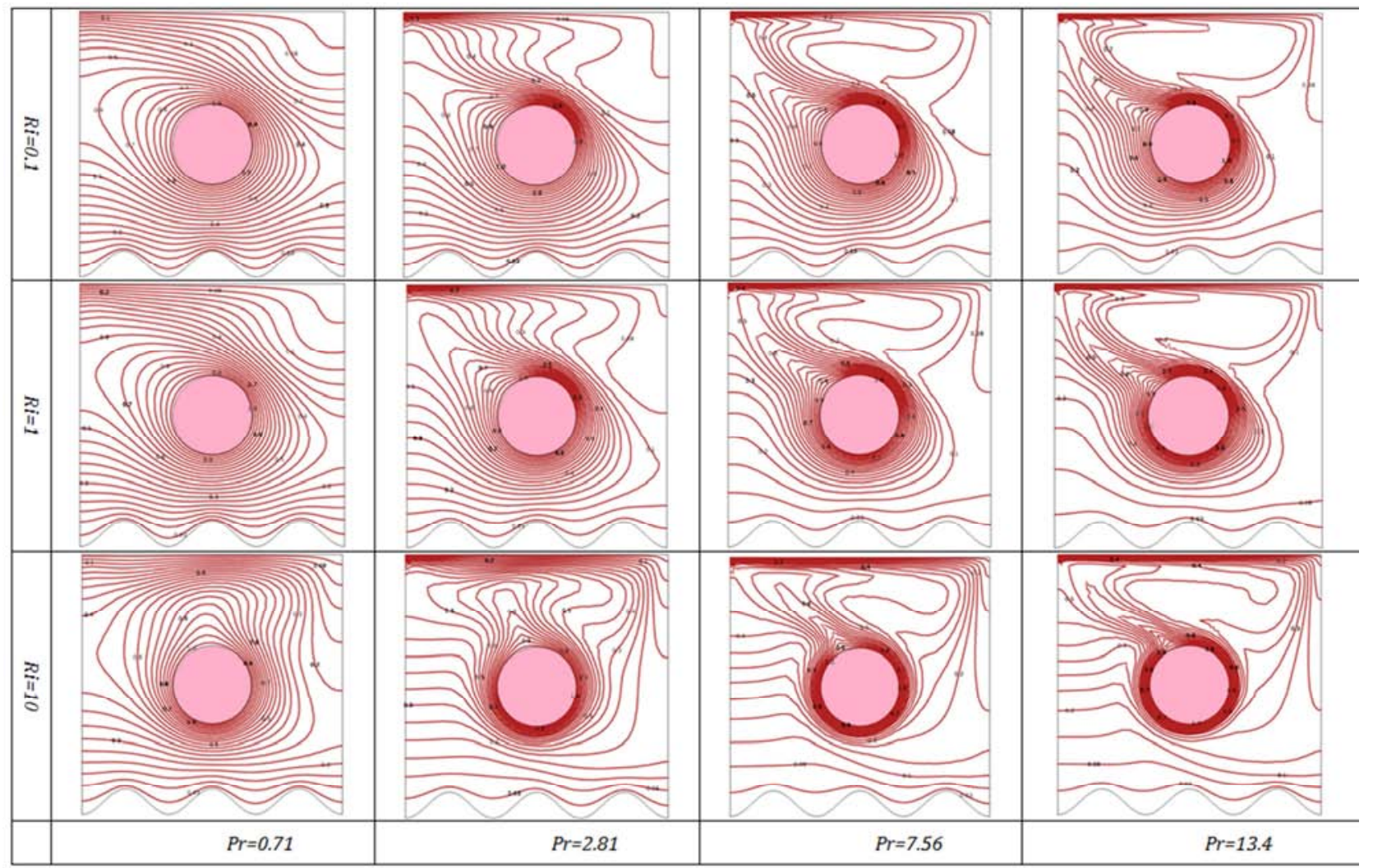

Figure 6. Isotherms for various values of Pr and Ri at $\mathrm{Re}=100, H a=20, A=0.05$.

The influence of $P r$ for various $R i$ on the temperature field are shown in figure 6. From figure it is clear that for $P r=0.71$ that is if the fluid is considered as air the buoyancy effect outweighs the effect of sliding wall and the heat transfer is mostly conductive. But with the increasing value of $\operatorname{Pr}$ the heat transfer by convection is being enhanced which is being reflected by the boundary layers near the heated body and upper vertical wall.

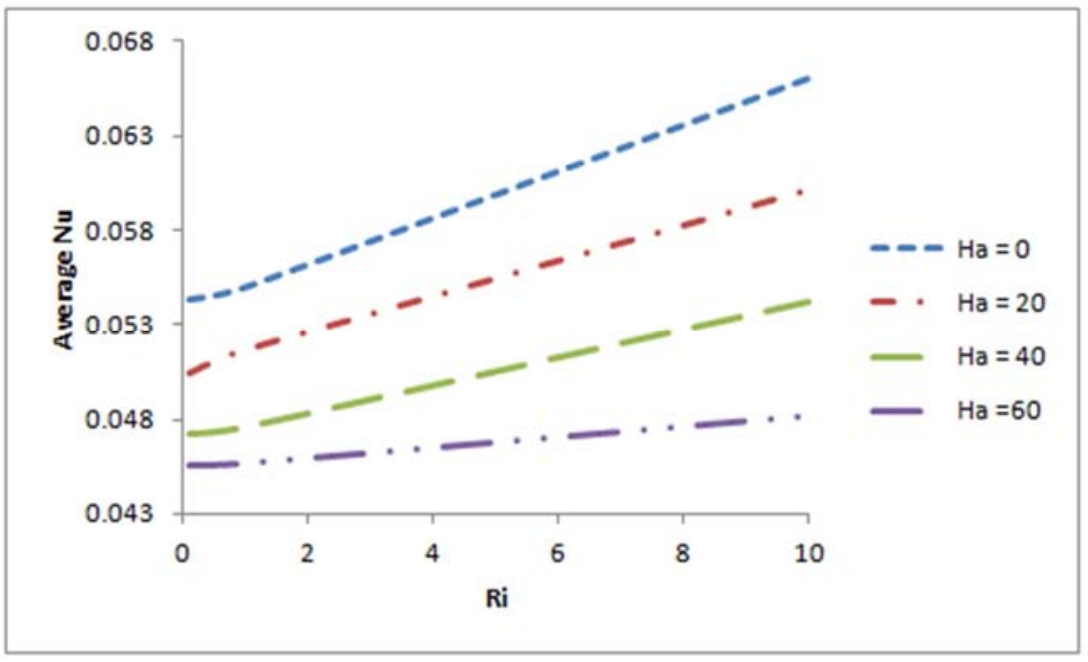

Figure 7. Average Nu along the heated wall for various $H a$ at $\operatorname{Re}=100, \operatorname{Pr}=0.71, A=0.05$. 


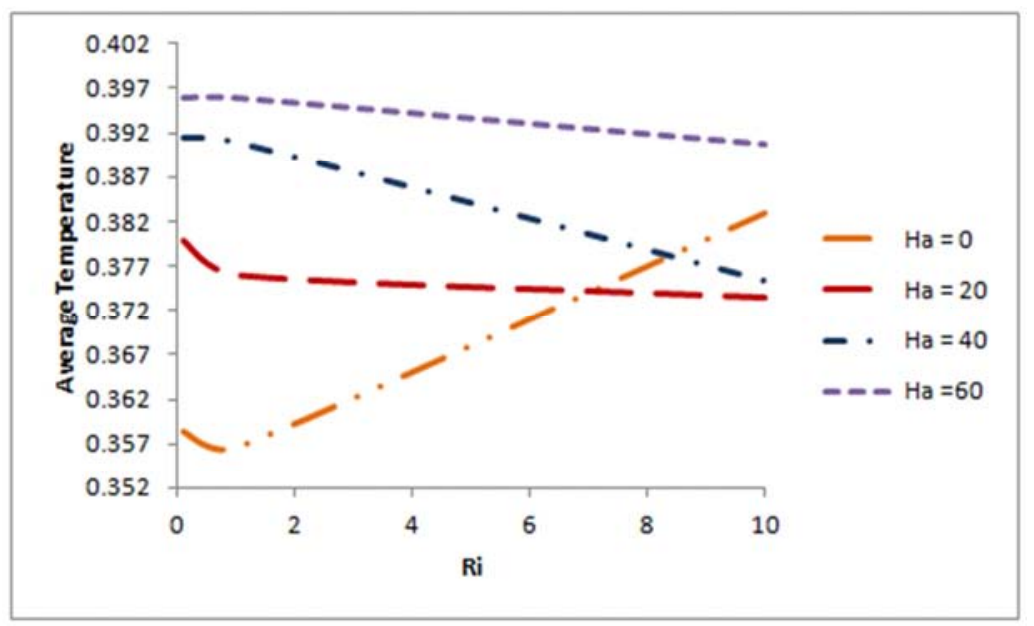

Figure 8. Average temperature of the enclosure for various Ha at $\operatorname{Re}=100, \operatorname{Pr}=0.71, A=0.05$.

The influence of Hartmann number on average Nusselt number at the hot surface of heated body is expressed in figure 7. From figure it is observed that the average Nusselt number increases with the increasing value of $R i$ for all values of $\mathrm{Ha}$. In absence of magnetic field $\mathrm{Nu}$ increases quickly but for increasing value of $\mathrm{Ha}$ this increment slows down gradually. On the other hand the average heat transfer is maximum for $H a=0$ and it decreases gradually for the increasing value of $\mathrm{Ha}$. The effect of magnetic field on average temperature for the variation of Richardson number is shown in figure 8 . From figure it is observed that, average temperature is maximum for highest value of $\mathrm{Ha}$ that is where magnetic field is strong. In absence of magnetic field, average temperature decreases in the forced convection dominated region but from mixed to natural convection dominated region it increases abruptly. It is also observed that for higher values of $\mathrm{Ha}$, average temperature decreases gradually with the increasing value of $R i$.

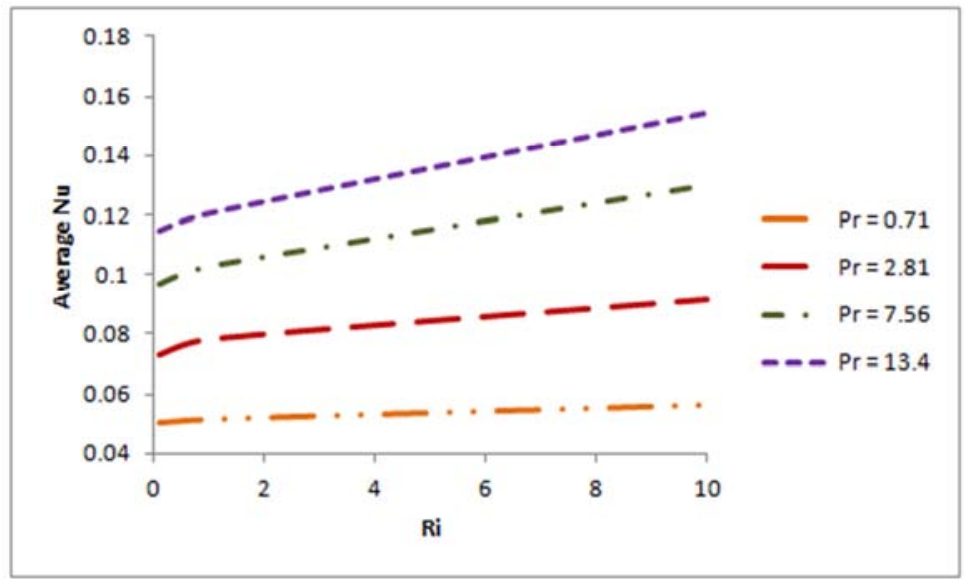

Figure 9. Average Nu along the heated wall for various $\operatorname{Pr}$ at $\operatorname{Re}=100, \operatorname{Pr}=0.71, A=0.05, \mathrm{Ha}=20$.

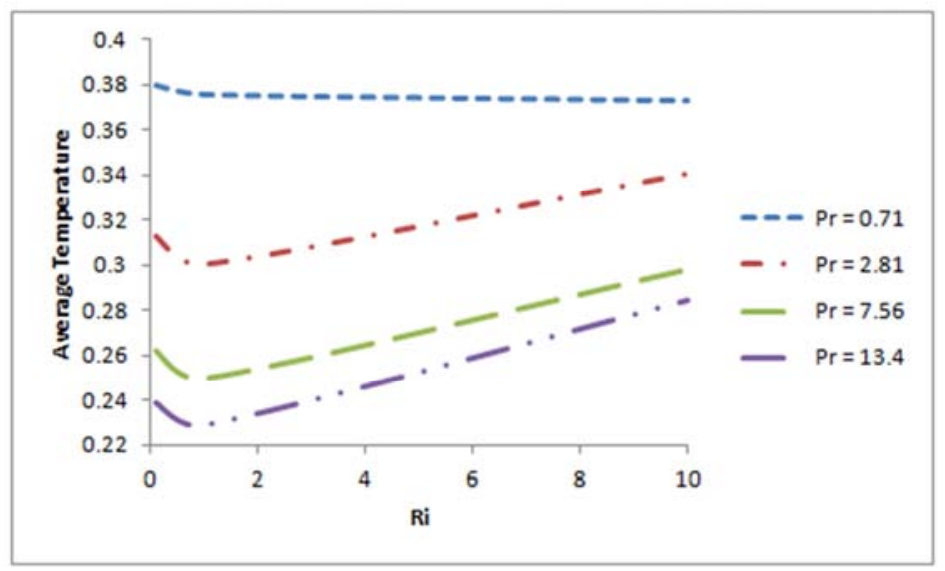

Figure 10. Average temperature of the enclosure for various $\operatorname{Pr}$ at $\operatorname{Re}=100, \operatorname{Pr}=0.71, A=0.05, \mathrm{Ha}=20$. 
The influence of Prandtl number on average Nusselt number at the hot surface of heated body is expressed in figure 9. From figure it is observed that the average Nusselt number increases with the increasing value of $R i$ for all values of $P r$. It is also observed that $\mathrm{Nu}$ is minimum for $\operatorname{Pr}=0.71$ and it is maximum for $\operatorname{Pr}=13.4$ i,e, heat transfer rate increases with the increasing value of Prandtl number. The influence of Prandtl number on average temperature of the fluid is expressed in figure 10. From figure it is observed that average temperature decreases with the increasing value of $P r$. It is also observed that, for $P r=0.71$ that is when the fluid is considered as air the average temperature remains almost constant for all values of $R i$ and for higher values of $P r$, it decreases in the forced convection dominated region but increases from mixed to natural convection dominated region.

\section{Nomenclature}

$\begin{array}{ll}c_{p} & \text { specific heat at constant pressure } \\ \mathrm{g} & \text { gravitational acceleration }\left[\mathrm{ms}^{-2}\right] \\ h & \text { convective heat transfer coefficient }\left[\mathrm{Wm}^{-2} \mathrm{~K}^{-1}\right] \\ k & \text { thermal conductivity of fluid }\left[\mathrm{Wm}^{-1} \mathrm{~K}^{-1}\right] \\ T & \text { dimensional temperature }[\mathrm{K}] \\ \Delta T & \text { dimensional temperature difference }[\mathrm{K}] \\ u, v & \text { dimensional velocity components }\left[\mathrm{ms}^{-1}\right] \\ U, V & \text { dimensionless velocity components } \\ p & \text { dimensional pressure }\left[\mathrm{Nm}^{-2}\right]\end{array}$

Greek symbols

$\alpha$
$\beta$
$v$
$\theta$
$\rho$

$$
\begin{aligned}
& \text { thermal diffusivity }\left[\mathrm{m}^{2} \mathrm{~s}^{-1}\right] \\
& \text { thermal expansion coefficient }\left[\mathrm{K}^{-1}\right] \\
& \text { kinematic viscosity }\left[\mathrm{m}^{2} \mathrm{~s}^{-1}\right] \\
& \text { non dimensional temperature } \\
& \text { density of the fluid }\left[\mathrm{kgm}^{-3}\right]
\end{aligned}
$$

\section{Conclusion}

A numerical investigation is made for steady-state, incompressible, laminar mixed convection flow in a square enclosure containing a heated obstacle. Results are obtained for wide range of parameters Richardson number (Ri), Hartmann number $(\mathrm{Ha})$ and Prandtl number $(\mathrm{Pr})$. The major findings have been summarized as follows:

The fluid flow and heat transfer are strongly dependent on Prandtl number. Heat transfer increases with the increasing value of Prandtl number.

Prandtl number plays an important role on fluid temperature. Fluid temperature decreases with the increasing value of Prandtl number.

Magnetic field plays a significant role on heat transfer and fluid flow. Heat transfer decreases with the increasing value of Hartmann number.

$x, y$
$X, Y$
$\left(C_{x}, C_{y}\right)$
$N u$
$P$
$P r$
$\mathrm{Gr}$
$D$

Cartesian coordinates
dimensionless Cartesian coordinates
circular obstacle center
average Nusselt number
dimensionless pressure
Prandtl number
Grashof number
Diameter of circular obstacle $[\mathrm{m}]$

Subscripts

$h$

heated

\section{References}

[1] Saha, L. K., Somadder, M. C., Salah Uddin, K. M. (2013) Mixed convection heat transfer in a lid driven cavity with wavy bottom surface. American Journal of Applied Mathematics 1 (5): 92-101.

[2] Nasrin, R. (2011) Mixed magnetoconvection in a lid driven cavity with a sinusoidal wavy wall and a central heat conducting body. Journal of Naval Architecture and Marine Engineering. DOI: 10.3329/ jname.v8il.6793.

[3] Rahman, M. M., Alim, M. A. (2010) MHD mixed convection flow in a vertical lid-driven square enclosure including a heat conducting horizontal circular cylinder with Joule heating. Nonlinear Analysis: Modeling and Control, Vol. 15, No. 2, 199-211.

4] Rahman M. M., Alim M. A., Mamun M. A. H (2009) Finite element analysis of mixed convection in a rectangular cavity with a heat conducting horizontal circular cylinder. Nonlinear analysis: Modeling and control, Vol.14, No. 2, 217-247.

[5] Hakan F. Oztop, Khaled Al salem, Loan Pop (2011) MHD mixed convection in a lid driven cavity with corner heater. International Journal of Heat and Mass Transfer 54 (2011) 3494-3504.

[6] R. YadollahiFarsani, B. Ghasemi (2011) Magnetohydrodynamic mixed convective flow in a cavity. World Academy of Science, Engineering and Technology. Vol. 5 2011-11-21.

[7] G. A. Sheikhzadeh, S. Mazrouei, M. Mahmooodi, Elham Safaeizadeh, S. E. Hashemi (2013) Effect of a Magnetic Field on Mixed Convection of a Nanofluid in a Square Cavity. Journal of Magnetics 18 (3), 321-325. 
[8] Parvin S., Nasrin R. (2012) Effects of Reynolds and Prandtl Number on Mixed Convection in an Octagonal Channel with a Heat Generating Hollow Cylinder. Journal of Scientific Research. J. Sci. Res. 4 (2), 337-348.

[9] N. A. Bakar, R. Roslan, A. Karimipour \& I. Hashim (2019) Mixed convection in lid-driven Cavity with inclined magnetic field. Sains Malaysiana 48 (2) (2019): 451-471.

[10] M. Muthtamilselvan, Deog Hee Doh (2014) Mixed convection of heat generating nanofluid in a lid-driven cavity with uniform and non-uniform heating of bottom wall. Applied Mathematical Modelling 38 (2014) 3164-3174.

[11] Oztop, H. F., Al-Salem K., Pop I. (2011) MHD mixed convection in a lid driven cavity with corner heater. International journal of heat and mass transfer 54 (2011) 3494-3504.

[12] Sivasankaran S., Sivakumar V., Prakash P. (2010) Numerical study on mixed convection in a lid driven cavity with nonuniform heating on both sidewalls. International journal of heat and mass transfer 53 (2010) 4304-4315.

[13] Roslan R., Saleh H., Hashim I., Bataineh A. S. (2014) Natural convection in an enclosure containing a sinusoidally heated cylindrical source. International journal of heat and mass transfer 70 (2014) 119-127.

[14] Parvin S., Nasrin R. (2011) Analysis of the flow and heat transfer characteristics for MHD free convection in an enclosure with a heated obstacle. Nonlinear Analysis: Modeling and Control, Vol. 16, No. 1, 89-99.

[15] Rahman M. M., Alim M. A., Saha S., Chowdhury M. K. (2008) A numerical study of mixed convection in a square cavity with a heat conducting square cylinder at different locations. Journal of Mechanical Engineering, Vol. ME 39, No. 2. 